\section{Skating on MTs}

$\int$ ynactin can skate along the length of a microtubule (MT), giving a boost to the attached dynein motor's forward progress, according to Tara Culver-Hanlon, Stephen King (University of Missouri, Kansas City, MOl, and colleagues.

Dynein is a poorly processive motor compared with others like kinesin. "It doesn't stay on the microtubule worth a hoot," says King. To be efficient, dynein needs dynactin, which has its own MT-binding domains. King's group found that dynactin had not only the wellknown CAP-Gly MT-binding domain, but also a neighboring basic domain that bound MTs.

When the group attached the domains to beads for single-particle tracking along MTs, the beads moved in distinctly different ways. CAPGly beads missing the basic sequence only swiveled around a relatively fixed point on the MT. Beads with only the basic domain or with both domains could swivel too, but they also skated the length of the MT track while still bound to it.

When the dynein motor was added to the mix, the basic domain alone or full-length dynactin increased the motor's processivity. The CAP-Gly domain alone, however, put the brakes on the motor's speed. The basic domain acts like a space-walking astronaut's tether, says King. "When dynein falls off, [the motor] can rebind and keep moving instead of floating off into the cytoplasm." ЈСB

Reference: Culver-Hanlon, T.L., et al. 2006. Nat. Cell Biol. doi: 10.1038/ncb1370.
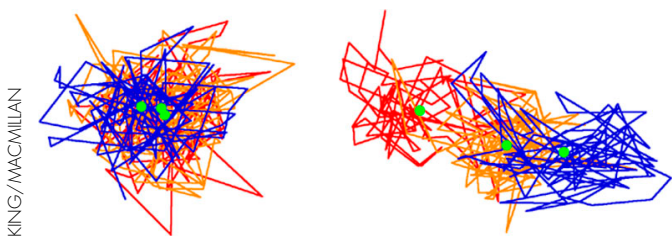

Mutant dynactin circles (left) rather than gliding along a microtubule (right).

\title{
Virus diverts checkpoint
}

T $\begin{aligned} & \text { uman cytomegalovirus (HCMV) inactivates a DNA damage response by } \\ & \text { booting two checkpoint proteins out of the nucleus, according to Miguel } \\ & \text { Gaspar and Thomas Shenk (Princeton University, Princeton, NJ). }\end{aligned}$

When DNA herpesviruses like HCMV replicate, the double-stranded ends of their genomes, which resemble breaks, can trigger a cell's DNA damage checkpoint pathway. Viruses have evolved several mechanisms to inactivate the checkpoint and proceed with replication, such as degrading an essential checkpoint component or using the block in cell cycle progression to their advantage.

Gaspar and Shenk now show that HCMV thwarts the checkpoint in a novel way-by trapping two essential checkpoint proteins, ATM and Chk2, in the cytoplasm. "This is the first time a virus has been found to antagonize a DNA damage checkpoint by mislocalization," notes Shenk.

The checkpoint proteins wind up hanging out with viral proteins in the cytoplasmic "virion assembly zone." Whether ATM and Chk2 get ferried out of the nucleus by the viral proteins or another viral protein blocks the checkpoint proteins' normal nuclear import remains to be answered.

Shenk says the HCMV studies could shed light on the poorly defined nuclear import mechanism-following his motto: "If a virus goes after it, it's probably important." JCB

Reference: Gaspar, M., and T. Shenk. 2006. Proc. Natl. Acad. Sci. USA. doi:10.1073/pnas.0511148103.

\section{Hungry for better memory}

1 growling stomach might lend a helping hand to memory, based on new work by Sabrina Diano, Tamas Horvath (Yale University School of Medicine, New Haven, CT), and colleagues.

The growling stomach produces the hormone ghrelin, which stimulates fat storage and appetite by binding neurons in the hypothalamus. But ghrelin also binds neurons in the hippocampus-a center for forming memories.

Horvath's team shows that ghrelin's hippocampal action promotes long-term potentiation and a higher synaptic density in the CAl region of the hippocampus. Both characteristics correlated with improved spatial memory and learning.

The authors injected rodents with ghrelin and used three behavioral tests that rely on hippocampal memory functions-exploring different arms of a plus-shaped maze and two foot-shock avoidance tests. In each, ghrelin improved memory performance in a dose-dependent manner. Performance was improved $20-30 \%$ at the highest dose, effectively turning C-grade mice into straight-A students. In a final test, ghrelin knockout mice showed little ability to recognize a novel object unless they got a shot of ghrelin.

Aged SAMP8 mice-a model for Alzheimer's disease-also showed improved memory performance with ghrelin dosing, and the authors propose ghrelin analogues as potential treatments for memory loss. Of course, over-eating and weight gain would be potential side effects.
Horvath says ghrelin represents a primitive system in which a gut hormone acts directly on the higher brain to change synaptic plasticity, and affect cognition. "If you are hungry, you need to be alert and aware of your environment," for example to help in finding the next meal. His group now plans to look for the same mechanism in humans. JCB

Reference: Diano, S., et al. 2006. Nat. Neurosci. doi: 10.1038/nn 1656.

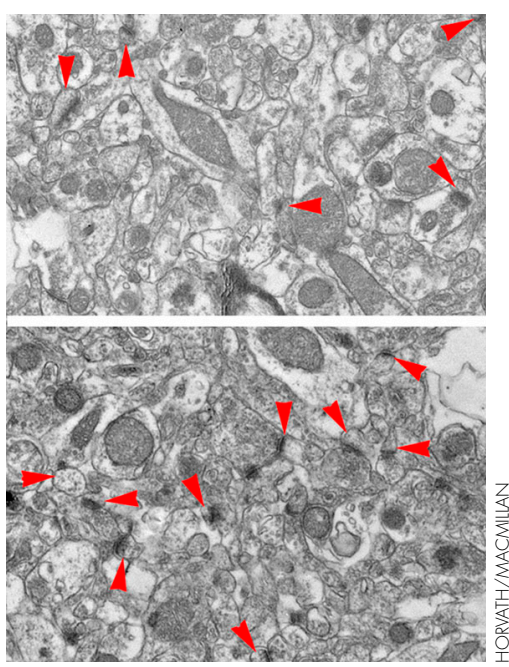

Gut hormone ghrelin (bottom) increases synapse density (arrowheads) and improves memory. 